Revue internationale de l'économie sociale

Recma

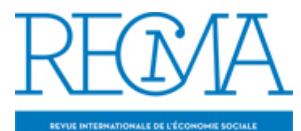

\title{
Hommage à Aliette Levecque (1942-2012), militante de l’économie sociale
}

\section{La rédaction}

Numéro 327, janvier 2013

URI : https://id.erudit.org/iderudit/1015144ar

DOI : https://doi.org/10.7202/1015144ar

Aller au sommaire du numéro

\section{Éditeur(s)}

Association Recma

\section{ISSN}

1626-1682 (imprimé)

2261-2599 (numérique)

Découvrir la revue

\section{Citer ce document}

La rédaction (2013). Hommage à Aliette Levecque (1942-2012), militante de l'économie sociale. Revue internationale de l'économie sociale, (327), 5-6. https://doi.org/10.7202/1015144ar 


\section{HOMMAGE À ALIETTE LEVECQUE (1942-2012), MILITANTE DE L'ÉCONOMIE SOCIALE}

(1) Benhamou J., Levecque A., La mutualité, Paris, PUF, Que-sais-je?, 1983.
(2) Lire "Une grande belle dame nous a quittés » de Paul Picard, sur www.ceges.org.
A liette Levecque est décédée le 16 octobre 2012. Fidèle membre du comité de rédaction de la Recma depuis 2002, elle a effectué toute sa carrière, de 1968 à 2003, à la Mutualité française, où ses talents d'organisatrice et ses qualités relationnelles ont été à l'origine de réalisations marquantes. Dès 1968, elle a mis en place et géré la bibliothèque, la photothèque et le service de documentation. Entre 1978 et 1990, elle était chargée des relations avec la presse, assurait le conseil en communication auprès des groupements mutualistes et organisait les événements (colloques, expositions, congrès). C'est durant cette période qu'elle a rédigé, avec Jean Benhamou, un "Que-sais-je? " sur la mutualitée ${ }^{(1)}$. La bibliographie sur la mutualité étant inexistante au début des années 80 , la lecture de ce petit ouvrage constituait alors, pour les apprentis-historiens, un préalable à toute incursion dans les archives mutualistes.

En 1990, Aliette a rejoint le secteur international de la Fédération nationale de la Mutualité française (FNMF), avec pour mission de promouvoir la mutualité à l'étranger et d'assurer le transfert du savoir-faire en matière d'assurance maladie et de protection sociale. Elle a effectué plusieurs missions dans les pays de l'Est et en Afrique, notamment au Sénégal pour la construction du centre de santé à Rufisque, une incontestable réussite. Chargée du secteur de l'économie sociale et solidaire à la FNMF avec Paul Picard, elle a participé à la création du Conseil des entreprises, employeurs et groupements de l'économie sociale (Ceges) ${ }^{(2)}$.

Aliette était également une militante du Parti socialiste, à Pantin et à Paris, une syndicaliste, fondatrice de la section CFDT à la FNMF, et une responsable mutualiste, siégeant au conseil d'administration de la Mutuelle d'entraide de la Mutualité française.

Depuis qu'elle avait fait valoir ses droits à la retraite, en 2004, elle n'avait pas pour autant déserté le terrain de l'économie sociale. Elle était tout d'abord l'auteure du très apprécié et précurseur Agenda de l'économie sociale, qui, à l'entête du Ceges et largement diffusé par courrier électronique, permettait chaque mois de lister toutes les manifestations en cours et à venir. Aliette s'inquiétait de savoir qui après elle reprendrait le flambeau de cette initiative, qu'elle savait utile à tous, salariés et militants, et dont la Recma avait doublé la publication depuis 2003 sous le juste titre de «l'Agenda d'Aliette Levecque ». La succession est assurée avec $L a$ Lettre de la Recma, diffusée chaque semaine auprès de tous ceux qui en font la demande.

Aliette participait, par ailleurs, au groupe Femmes du Mouvement européen de France, association militant en faveur de la construction européenne 
dans une perspective fédérale. Membre actif d'une association d'aide au développement et aux échanges Nord-Sud pour l'envoi de médicaments, la prévention du sida, la création d'un centre de santé et l'envoi de bourses pour les jeunes, elle avait été élue "Reine de tous les Européens " par les habitants du village de Mempassem, au Togo.

Aliette était l'unique représentante de la branche des mutuelles santé au comité de rédaction de la Recma, traditionnellement composé de chercheurs et d'acteurs des mouvements de l'économie sociale. La disparition de cette militante aussi chaleureuse qu'efficace laisse un grand vide auprès de tous ceux qui l'ont côtoyée.

La rédaction 